\title{
The stimulus prefix is not irrelevant and is redundant in different ways
}

\author{
JOHN C. JAHNKE and RAY E. BOWER \\ Miami University, Oxford, Ohio \\ and \\ ROBERT A. BJORK \\ University of California, Los Angeles, California
}

\begin{abstract}
The present study examined the stimulus prefix effect of immediate recall under conditions in which the prefix element was defined explicitly as being an irrelevant item that should be ignored, or, alternatively, as an item to be remembered and recalled. Memory was poorer in the latter case. Also examined were the effects of three types of redundancy relation between the prefix and target elements; one of these, interstring redundancy (same prefix precedes each target string), was found to improve recall in a relative sense, and another, conceptual redundancy (prefix and target items belong to the same conceptual category), was found to degrade it. Thus, that part of the conventional stimulus prefix effect attributable to redundancy, per se, can be viewed as a composite of at least two opposing factors. Finally, the independent variables of the present study were interpreted to impose different information-processing requirements on the subject, and an account of these requirements was offered.
\end{abstract}

Interest in the effect of a redundant stimulus element on recall dates from the work of Dallett $(1964,1965)$. Apparently generalizing from a procedure first introduced by Conrad (1958, 1960), Dallett presented span-length control strings of the decimal digits 1-9 for immediate recall. Experimental strings were comparable, except that each memory series was prefixed by the digit zero. The subject was under no obligation to recall the prefix element and, in fact, was told to disregard it. However, the effect of the prefix was to reduce significantly the recall of the memory series, a surprising result at the time.

The prefix element in studies of this sort has been described as being irrelevant-irrelevant in that the prefix was to be disregarded; however, because performance is poorer in the prefixed than in the unprefixed condition, two current interpretations of the stimulus prefix effect (Jahnke \& Perez, 1981; Neisser, Hoenig, \& Goldstein, 1969) have assumed that the prefix element is relevant, in the sense that it cannot be ignored. Yet it is not known presently to what degree this is so. It is possible, for example, that only some subjects fail to ignore the stimulus prefix only on some proportion of the experimental trials.

One test of this notion, and one embraced in this study, is to contrast conditions in which the prefix is to be dis-

This study was supported in part by a grant from the Faculty Research Committee of Miami University. Scott Cone and Gina Klenkar assisted capably with the collection and analysis of data, and the comments of three anonymous reviewers are gratefully acknowledged. Requests for reprints should be sent to J. C. Jahnke, Department of Psychology, Miami University, Oxford, OH 45056. regarded with ones in which the prefix is explicitly defined as relevant-to be given attention and recalled. Two studies in the extant literature have included such conditions in the conventional paradigm in which the prefix and target items were digits and the same digit, zero, preceded each string. Dallett (1964) used completely correct recall as the dependent measure and found nonsignificantly poorer recall in the relevant than in the irrelevant prefix condition. A second study (Jahnke, Nowaczyk, \& Wozniak, 1976) was designed to examine the effect of multiple affixes and used percentage correct recall as the dependent measure. In this case, significantly greater impairment in performance was found when the prefix was relevant than when it was irrelevant.

The stimulus prefix element also has been described as being redundant, in that the identity of the prefix was known to be the same on all experimental trials. Consideration of the method, however, shows that the prefix in conventional studies is redundant in two different senses. First, it is redundant in the sense of having conceptual redundancy: the prefix and target elements are members of the same conceptual category. Second, it is redundant in the sense of being known in advance and having what we call "interstring redundancy": the prefix is held constant throughout a block of trials and the subject is instructed to that effect. There is a third type of prefix redundancy not found in typical studies of the stimulus prefix effect, but one which, in principle, could be utilized. We call this form of redundancy "prefix-target redundancy": the prefix word of one string is permitted to be the target word of another string, and vice versa. Briefly put, the present study is an attempt to determine whether, and in 
what ways, redundancy and relevancy of the prefix contribute to its disruptive effect on recall.

\section{METHOD}

\section{Subjects}

The subjects were 25 Miami University students (12 male and 13 female) enrolled in introductory psychology courses. Participation in this experiment was one available option for completing a service requirement in those courses. None of the subjects had previously served in research concerned with immediate memory. The data from 5 additional subjects were discarded because the subjects failed to follow instructions.

\section{Materials}

The memory series were 110 seven-word strings. Each string was a random permutation drawn from the pool of animal names: bull, deer, fox, goat, hippo, leopard, panther, rabbit, skunk, and zebra. Strings were constructed so that a given word never occupied the same serial position in consecutive strings and so that the same two reportable words did not occupy the first two or last two serial positions in consecutive strings. In addition, in all but four instances owed to experimenter error, the last item in one string was not the first reportable item in the next.

\section{Design}

All subjects served in each of 11 experimental conditions; each condition was represented by a block of 10 strings, the first 2 of which were considered as practice, and the data from which were discarded. One of the 11 conditions was a control comprising only the seven-word strings of animal names. Each of the remaining conditions included similar seven-word strings, except that each was prefixed by the name of an animal or a vehicle. Of these 10 conditions, 8 represented the factorial combination of three independent variables, each with two levels: (1) prefix relevancy (the prefix was either irrelevant-to be ignored and not recalled-or was relevant - to be given attention and recalled), (2) conceptual redundancy (the prefix was either the name of an animal or a vehicle), and (3) interstring redundancy (for a given condition, the prefix was always the same word or was different for each string). The remaining 2 conditions were included in an attempt to examine the effect of the third type of redundancy, prefix-target redundancy. In both of these conditions, the prefix was an animal name. For a given string, the animal prefix was permitted to become a member of the memory series of another string, and vice versa. In 1 of these 2 conditions, the prefix was relevant (as defined above), and in the other, irrelevant.

In those conditions in which the prefix was held constant within a block and was an animal name, it was always the word "mule." When held constant and the name of a vehicle, it was the word "ship." When the prefixes were different for each string within a block (condition), the prefixes were randomly assigned without replacement to strings from the pool including the words ape, camel, donkey, giraffe, lamb, mule, rhino, sheep, squirrel, and wolf, or from the pool including the words bike, cart, jeep, jet, scooter, ship, taxi, tractor, trailer, and wagon. Both pools contained five one-syllable and five two-syllable words, and the average frequencies of the words in each pool, as indexed by the Battig and Montague (1969) norms, were approximately equal.

\section{Procedure}

Subjects were tested in groups of 6-8. Testing consisted of listening to the tape-recorded strings, with elements of each list spoken at a rate of two per second in a female monotone voice. When present, the prefix word was spoken in rhythm with the target series. A silent interval of about $16 \mathrm{sec}$ followed each series, during which written, ordered recall was attempted. To ensure that this was adequate time for recall, subjects were instructed to write only the first two letters of each target item.
The 11 conditions were presented to subjects in one of four different orders. The ordering of conditions was random, with the exception that two of the sequences began with an irrelevant prefix and two began with a relevant prefix. Before each condition was presented, subjects were apprised of whether or not there was a prefix, and, if so, its nature (animal or vehicle; constant or variable, and the specific word if constant; and whether the prefix was to be reported or ignored). Subjects were instructed to record the target items in the order in which they had been presented, starting with the first word and so on in order and to leave blank the location of any word they could not remember. Items were scored as correct only if given in the proper serial position on specially prepared answer sheets. Subjects were also instructed not to write anything while a string was being presented.

\section{RESULTS}

Eleven conditions were established for this experiment in order to test the effects of prefix relevancy and three kinds of prefix redundancy. Both planned and unplanned statistical comparisons among these conditions were anticipated. First, it was desired to compare all 10 prefix conditions, regardless of whether the prefix was to be recalled or not, with the conventional, unprefixed control. Table 1 shows that mean proportion error for the seven target elements was higher in each of the 10 prefix conditions than in the control, and a planned comparison gave $\mathrm{F}(1,240)=29.98, \mathrm{MSe}=24.13, \mathrm{p}<.05$, the rejection region used throughout this report. Thus, as anticipated, there was a reliable effect of the stimulus prefix, measured in terms of the overall difference in performance between prefixed and control strings. Although not presented here, the serial-position curves for the prefix conditions were elevated above, and essentially parallel to, that for the unprefixed control condition (cf. Jahnke \& Perez, 1981). As will now be seen, the magnitude of the increase in error rate depended on the nature of the prefix condition.

A second set of comparisons involved the eight prefix conditions that comprise the factorial combination of three variables, (1) relevancy, (2) conceptual redundancy, and (3) interstring redundancy (constant/varied prefixes), as shown in Table 1. A related-measures $2 \times 2 \times 2$ factorial

Table 1

Mean Proportion Error in the Ordered Recall of Seven-Word Strings (Animal Names) as a Function of Experimental Condition

\begin{tabular}{lccccc}
\hline & \multicolumn{4}{c}{ Prefix Category } \\
\cline { 3 - 6 } & & \multicolumn{2}{c}{$\begin{array}{c}\text { Vehicle } \\
\text { (No Conceptual } \\
\text { Redundancy) }\end{array}$} & \multicolumn{2}{c}{$\begin{array}{c}\text { Animal } \\
\text { (Conceptual } \\
\text { Redundancy) }\end{array}$} \\
$\begin{array}{l}\text { Rrefix } \\
\text { Relevancy }\end{array}$ & $\begin{array}{c}\text { No-Prefix } \\
\text { Control }\end{array}$ & $\begin{array}{c}\text { Constant } \\
\text { Prefix }\end{array}$ & $\begin{array}{l}\text { Varied } \\
\text { Prefix }\end{array}$ & $\begin{array}{c}\text { Constant } \\
\text { Prefix }\end{array}$ & $\begin{array}{c}\text { Varied } \\
\text { Prefix }\end{array}$ \\
\hline $\begin{array}{l}\text { Irrelevant } \\
\text { (Not-to-be }\end{array}$ & & & & & \\
$\begin{array}{l}\text { Reported) } \\
\text { Relevant } \\
\text { (to-be }\end{array}$ & .41 & .48 & .45 & .48 & $.50(.49)^{*}$ \\
Reported) & - & .50 & .55 & .50 & $.60(.54)$ \\
\hline
\end{tabular}

*The values in parentheses denote error proportions when there was prefix-target redundancy across trials. 
analysis of variance for these eight conditions showed that the main effect of each variable listed above was significant $[\mathrm{F}(1,24)=13.47, \mathrm{MSe}=38.01 ; \mathrm{F}(1,24)=5.64$, $\mathrm{MSe}=14.53$; and $\mathrm{F}(1,24)=7.31$, $\mathrm{MSe}=19.78$, respectively]. Performance was significantly poorer when the prefix was relevant (to be included in recall), was better with interstring redundancy (constant prefixes), and was poorer with conceptual redundancy (animal prefixes).

The only additional significant effects in this analysis were two first-order interactions, those between interstring redundancy and relevancy $[\mathrm{F}(1,24)=10.59, \mathrm{MSe}=$ 23.70] and between interstring redundancy and conceptual redundancy $[F(1,24)=4.50, \mathrm{MSe}=20.57]$. Separate Newman-Keuls analyses examined the nature of these interactions. First, when the prefix was relevant, a variable prefix added significantly to the inhibitory effect on recall. Second, when the prefix and target elements were from the same conceptual (animal) category, a variable prefix again added significantly to its inhibitory effect on recall.

It also follows that the effect of a variable prefix should increase over successive trials, particularly when the prefix is relevant and is encoded. (The number of encodings from which recall can occur increases with trials.) This expectation was tested by comparing performance on the first three trials within a block (this analysis includes the two practice trials) with that on the last three triais. Wilcoxon matched-pairs signed-ranks tests showed that recall of the target elements declined significantly over trials in both the relevant/varied/animal and the relevant/varied/vehicle conditions $[\mathrm{T}=52.5, \mathrm{~N}=21$, and $\mathrm{T}=74, \mathrm{~N}=24$, respectively].

When the prefix is irrelevant, however, it may fail to be encoded on at least some of the trials; for such conditions, this increased effect of the variable prefix with trials would be diminished or nonexistent. Similarly, when the prefix is relevant but constant, vocabulary size is increased only by a single item; furthermore, the constancy of the prefix would work against any disruptive effect. Such factors might become increasingly prominent later in a block of trials, and, in all these cases, recall might be expected to remain relatively stable, or even improve, within a block of trials. Wilcoxon tests performed separately for each of these other conditions showed a small but nonsignificant improvement in performance over the course of the 10-trial blocks in five of the six contrasts. Only the irrelevant/constant/animal (conventional prefix) condition showed a small loss, one that appears to be attributable to relatively good performance on the first trial.

A third set of comparisons asked whether or not prefixtarget redundancy contributed additionally to the effect of the stimulus prefix (see the values in parentheses in Table 1). One comparison contrasted the conventional (irrelevant/constant/animal) prefix condition with the two in which the prefix element may become a target element in a subsequent string (and vice versa) within the same block of trials. Of these latter two, one required recall of the prefix, while the other did not. Because both of these conditions permitted the possibility of considerable confusion between prefix and target elements over a series of trials, performance was expected to be poorer in these conditions than in the conventional stimulus prefix condition. Although the differences shown in Table 1 are not large in absolute magnitude, they were in the predicted direction; a planned comparison showed that performance was significantly poorer in the two conditions of targetprefix redundancy than in the conventional prefix condition $[F(1,48)=6.97, \mathrm{MSe}=32.36]$. This relation was quite consistent across subjects; 18 of the 25 showed the expected effect $(p<.05)$

A second kind of comparison contrasts each of the two prefix-target redundancy conditions with a prefix condition that is similar except for prefix-target redundancy. For example, the condition that includes prefix-target redundancy (and thus, also, conceptual redundancy) and for which the varied prefixes are relevant can be compared with the condition with only conceptual redundancy and varied, relevant prefixes. Related-measures $t$ tests showed that the effect of prefix-target redundancy, over and above that of conceptual redundancy alone, depended on whether or not the prefix was to be recalled. When it was to be recalled, prefix-target redundancy improved recall $[\mathrm{t}(24)=2.68, \mathrm{SE}=1.15]$. It should be noted, however, that when there was prefix-target redundancy, prefixes and target items were from the same set of animal names and the size of the pool from which recall nominally occurred was 10 ; when there was only conceptual redundancy, however, prefixes and targets were from different sets of animal names and the size of the pool was 20 . Thus, increased vocabulary size (and cue overload) was associated with poorer recall in this case.

On the other hand, when the prefix was not to be recalled, prefix-target redundancy did not improve performance significantly in relation to that associated with conceptual redundancy alone $[\mathrm{t}(24)=0.48, \mathrm{SE}=1.25]$, even though vocabulary sizes were again different in the two comparison groups. The difference between these two comparisons shows that the impact of increased vocabulary size is negligible when the varied prefixes need not be recalled. One interpretation of this difference is that when the prefix is irrelevant, it can sometimes be ignored; when this is so, the smaller vocabulary associated with prefix-target redundancy provides less advantage than it otherwise would. In effect, the difference in vocabulary sizes is diminished, relative to the case in which the prefix is required in recall.

Finally, the difference between the two conditions of prefix-target redundancy closely approached significance [related-measures $\mathrm{t}(24)=1.98, \mathrm{SE}=1.46$ ]; recall was better when prefixes were to be disregarded.

\section{DISCUSSION}

\section{The Effects of Relevancy}

One purpose of the present experiment was to determine whether or not an explicitly "relevant" stimulus pre- 
fix degraded recall by an amount over and above that associated with an explicitly "irrelevant" stimulus prefix. Present data showed clearly that performance was poorer when the subjects were instructed to remember and recount the (relevant) stimulus prefix than when they were instructed to ignore the (irrelevant) prefix. One interpretation of this outcome is that some subjects in the irrelevant-prefix condition are able to ignore the prefix element. A second possibility is that output interference associated with the act of recall accounts, at least in part, for this difference (cf. Jahnke et al., 1976).

In addition, present data remind us that even when subjects are instructed to ignore the stimulus prefix, performance is not as good as when unprefixed control strings are the memory stimuli. Thus, it has been argued that the "irrelevant" stimulus prefix is actually relevant, in the sense that it is encoded as part of the stimulus, and in effect increases the length of the functional memory series. Evidence in favor of such a proposal includes instances in which the prefix actually was an intrusion given in recall. One might suppose that such cases would not exist, that even those subjects who, contrary to instruction, failed to ignore the prefix would edit out such obvious errors in recall. Inspection of the raw data of the present study showed no instances in which a prefix word from the vehicle category was included in recall of the (animal) target items. However, our data did show that when both the prefix and target items were members of the animals category, 41 such errors (out of 800 opportunities) did occur. Twelve of the 25 subjects gave at least 1 such intrusion, and most such intrusions ( 34 of the 41 ) occurred in the two conditions with prefixes that varied from trial to trial within a condition. Of these two conditions, 9 subjects made 23 such errors in the condition in which prefixes were relevant, and 6 subjects made 11 such errors when the prefix was irrelevant. Only 3 subjects gave more than 2 such intrusions in any one condition (relevant/varied/animal), and the largest number made by any subject in a given condition was 7 .

As noted earlier, two current interpretations of the stimulus prefix effect have adopted the assumption that the stimulus prefix is relevant (i.e., cannot be ignored, even when the subject is instructed to do so). For example, Neisser et al. (1969) have argued that, because there is no inherent meaning to a string of random digits, items are encoded by means of a Gestalt organizational process based on rhythmic grouping. This is an interpretation based on a preattentive process, one in which the prefix and target elements are obligatorily grouped as a single utterance. Because the memory series is now one element (the prefix) longer, recall of the target elements is degraded.

A second interpretation (Jahnke \& Perez, 1981) is based on cue-overload theory (M. J. Watkins, 1979; M. J. Watkins \& O. C. Watkins, 1976; O. C. Watkins \& M. J. Watkins, 1975). Cue-overload theory asserts that recall of a series of items is mediated by retrieval cues and that the probability of recall of an item is inversely proportional to the number of target items that share its retrieval cue. On this view, a stimulus prefix that is structurally, physically, or semantically related to the target elements may readily share with them a common retrieval cue (see M. J. Watkins \& O. C. Watkins, 1976). In this way, recall is poorer for prefixed than for unprefixed control strings. Although the perceptual organization interpretation of Neisser et al. (1969) specifies that preattentive processes underlie the stimulus prefix effect, both the present and earlier data (Jahnke \& Perez, 1981) have shown that postcategorical, semantic processes also are involved; in neither case, however, is it necessary to assume that the prefix is encoded by all subjects on all trials.

\section{The Effects of Redundancy}

Of the two forms of redundancy involved in typical studies of the stimulus prefix effect, conceptual redundancy (prefix and target elements are members of the same conceptual category) served to worsen recall. Interstring redundancy (same prefix used on all trials within a condition), on the other hand, served to improve recall. Both effects are reasonable when considered in light of the respective loads they place on memory.

Conceptual redundancy increases intrastring stimulus similarity and, therefore, the likelihood of cue overload. Thus, recall should be poorer when there is conceptual redundancy. (In the present study, this effect was marked only when the identity of the prefix element was not known in advance, although large effects of conceptual redundancy when the prefix was known in advance have been observed previously with digit, letter, and word stimuli; Jahnke \& Perez, 1981.)

Interstring redundancy, alternatively, appears to lighten memory load and, perhaps, accomplishes this in more than one way. Firstly, when the prefix is always the same element over a block of trials, the fact that its identity is known in advance may provide for more efficient encoding and retrieval of the target series. Secondly, even when the subject has been instructed to ignore the stimulus prefix, the subject is obviously not (always) able to do so; present data suggest that this may be particularly the case for conditions in which variable prefixes were the names of animals. The size of the stimulus pool from which recall could occur and the probability of guessing incorrectly are increased in such circumstances; as a consequence, error rates are increased in relation to the condition in which the prefix element is always the same. Lastly, and perhaps most importantly, when prefixes are variable, the likelihood of cue overload is increased, because the number of different elements sharing the conceptual category retrieval cue will increase as one stimulus series follows another, each with a new and different prefix from that category.

In view of these considerations, the typical stimulus prefix effect may best be understood as a composite of at least two separate and opposing effects of redundancy: a positive effect on recall because the prefix is the same element throughout a series of trials, and a negative ef- 
fect because the prefix and target elements are members of the same conceptual category.

\section{Cue Overload and Retrieval Inhibition}

The interpretation of the present data in terms of cueoverload principles has been advanced above. In this view, prefix constancy was seen to facilitate performance by reducing to a minimum the number of items that share a common retrieval cue. Furthermore, prefix relevancy and conceptual similarity between prefix and target items were seen to disrupt performance by increasing that number.

It should be noted, however, that another current concept, retrieval inhibition (e.g., Bjork \& Geiselman, 1978; Kato, 1985), may also be relevant to the interpretation of the present data. A retrieval-blocking (retrievalinhibition) notion argues that the elicitation of strong nontarget items (which may be readily recognizable as such) can block retrieval access to weaker target items. Thus, constant prefixes, even when irrelevant, become strongly associated to the experimental context. They are, therefore, readily retrieved, which may block or slow down access to target items.

\section{A Processing Analysis of the Stimulus Prefix Effect}

The various factors outlined above can be viewed as prefix-induced additions to a subject's processing loadsome contributing to processing load during storage, and some contributing to processing load during retrieval.

First among the factors contributing to memory load is the presence of a prefix. On the assumption that the prefix is encoded by at least some subjects on some trials, even if instructions have been given to disregard it, memory load will be greater for a prefixed string than an unprefixed control. This factor, of course, has been implicated in most, if not all, previous research on the stimulus prefix (e.g., Crowder, 1967; Dallett, 1964; Neisser et al., 1969). However, when the prefix is relevant, as in some conditions of the present experiment, all subjects can be presumed to attempt to encode the prefix on all trials. For this reason, storage load can be expected to be greater for conditions with a relevant, as opposed to an irrelevant, prefix. Finally, if the relevant prefix varies from trial to trial, the effort required to process the prefix is presumed to be greater. The entries in Column 1 of Table 2 reflect the foregoing arguments. All conditions of the present experiment are included in Table 2 except the two that combine conceptual and prefix-target redundancy. These have been omitted because of additional complications that arise when a prefix becomes a target element and vice versa.

Conceptual similarity is considered here to be a second factor contributing to storage load. When the prefix is from the same conceptual category as the target items, there is an additional contribution to memory load that presumably arises primarily from cue overload (as outlined previously). However, when the prefix is concep- tually similar but is constant (e.g., the same animal name on each trial in the present experiment), cue overload will be less than when it is varied, and perhaps will be no greater than when the prefix is from a different conceptual category. Thus, one might expect conceptual similarity to have an effect only when the prefixes vary from trial to trial. The data of the present study gave evidence of such an interaction; conceptually similar prefixes were more difficult when they were varied, and conceptual similarity otherwise had no significant effect. This interaction is identified in Column 2 of Table 2, where only the two varied/animal conditions are marked with a substantial contribution from the conceptual similarity factor.

A third factor contributing to storage load-prefix variability -is also represented in Column 2 of Table 2. Whereas a constant prefix added only a single new encoded element to the set $(n=10)$ from which recall would most likely occur in the present case, variable prefixes doubled in size the pool of encodings nominally available for recall from 10 to 20 . Prefix variability, however, should interact with prefix relevancy; irrelevant prefixes are unlikely to be retained across trials. For this reason, only the two relevant/varied conditions include a contribution from this factor. The entries in Column 2 assume that the similarity factor is more important than is the variability factor.

A fourth factor is considered to impact processing load during retrieval and is associated with editing processes during recall. If the prefix has been encoded but is not to be emitted in recall, processing resources that would otherwise have been directed to retrieval are devoted to the elision of the prefix. Such a factor is expected to influence only those conditions for which the prefix is irrelevant, as defined above. Contributions from this source are shown in Column 3 of Table 2 . It will be recalled, however, that vehicle prefixes were never given as overt intrusions; therefore, the contribution from editing shown for the two irrelevant/vehicle conditions is arguable.

Another factor, output interference, is also considered to add to processing load during retrieval. Column 4 in Table 2 reflects the influence of two different types of interference at output: (1) the disruptive effect of the prefix when it is relevant and, therefore, is output in recall (cf. Murdock, 1963; Tulving \& Arbuckle, 1963); and (2) the potential retrieval blocking of target items attributable to the presence of a strong (constant) prefix item in memory. The entries in Column 4 assume that the output interference of the first type is greater in the relevant/varied condition than in the relevant/constant conditions, owing to the need to output a different prefix on each trial in the varied conditions.

Table 2 presents in schematic form the prefix-induced disruptive effects in the conditions of most interest in the present experiment. By making the simplifying assumptions that each plus sign entered in Table 2 contributes approximately equally to the disruption of recall and can be summed algebraically, the total contribution to any condition is shown in Column 5. Each of these values can 
Table 2

Presumed Disruptive Effects Owing to a Stimulus Prefix

\begin{tabular}{|c|c|c|c|c|c|c|}
\hline \multirow[b]{2}{*}{$\begin{array}{l}\text { Nature of the } \\
\text { Stimulus Prefix }\end{array}$} & \multicolumn{2}{|c|}{$\begin{array}{c}\text { Processing Load } \\
\text { During Storage }\end{array}$} & \multicolumn{2}{|c|}{$\begin{array}{l}\text { Processing Load } \\
\text { During Retrieval }\end{array}$} & \multirow{2}{*}{\multicolumn{2}{|c|}{$\begin{array}{l}\text { Observed } \\
\mathrm{P} \text { (error) }\end{array}$}} \\
\hline & $\begin{array}{c}\text { Prefix } \\
\text { Encoding }\end{array}$ & $\begin{array}{c}\text { Across-Trials } \\
\text { Similarity- } \\
\text { Set Size }\end{array}$ & $\begin{array}{c}\text { Response } \\
\text { Editing }\end{array}$ & $\begin{array}{c}\text { Output } \\
\text { Interference }\end{array}$ & & \\
\hline No Prefix (Control) & 0 & 0 & 0 & 0 & 0 & .41 \\
\hline Irrelevant Constant Vehicle & + & 0 & + & + & 3 & .48 \\
\hline Irrelevant Constant Animal & + & 0 & + & + & 3 & .48 \\
\hline Irrelevant Varied Vehicle & + & 0 & + & 0 & 2 & .45 \\
\hline Irrelevant Varied Animal & + & ++ & + & 0 & 4 & .50 \\
\hline Relevant Constant Vehicle & $+t$ & 0 & 0 & ++ & 4 & .50 \\
\hline Relevant Constant Animal & ++ & 0 & 0 & ++ & 4 & .50 \\
\hline Relevant Varied Vehicle & +++ & + & 0 & ++ & 6 & .55 \\
\hline Relevant Varied Animal & ++ & ++ & 0 & ++ & 8 & .60 \\
\hline
\end{tabular}

Note-The extent of the presumed effect is indexed by the number of pluses.

be compared with the corresponding obtained proportion error, which is shown in Column 6. As can be seen, there is good agreement between expectation and outcome. Naturally, however, the present data cannot be considered to validate this post hoc analytical approach. The obtained good correspondence of "theory" and data does demonstrate that such a storage-load/retrieval-load analysis is consistent with the present results. In that sense, the analysis provides a potentially useful framework in terms of stimulating further analysis and experimentation.

\section{REFERENCES}

Battig, W. F., \& Montague, W. E. (1969). Category norms for verbal items in 56 categories: A replication and extension of the Connecticut category norms. Journal of Experimental Psychology Monographs, 80(3, Pt. 2).

Bjork, R. A., \& Geiselman, R. E. (1978). Constituent processes in the differentiation of items in memory. Journal of Experimental Psychology: Human Learning \& Memory, 4, 347-361.

ConRaD, R. (1958). Accuracy of recall using keyset and telephone dial, and the effect of a prefix digit. Journal of Applied Psychology, 42, 285-288.

ConRad, R. (1960). Very brief delay of immediate recall. Quarterly Journal of Experimental Psychology, 12, 45-47.

Crowder, R. G. (1967). Prefix effects of immediate memory. Canadian Journal of Psychology, 21, 450-461.

DalletT, K. M. (1964). Effects of a redundant prefix on immediate recall. Journal of Experimental Psychology, 67, 296-298.
Dallett, K. M. (1965). "Primary memory": The effects of redundancy upon digit repetition. Psychonomic Science, 3, 237-238.

JahNKE, J. C., NowaCzyK, R. H., \& WozNiak, W. (1976). Stimulus redundancy and immediate recall. Memory \& Cognition, 4, 357-360.

Jahnke, J. C., \& Perez, W. A. (1981). Semantic encoding and the stimulus prefix effect. Joumal of Verbal Leaming \& Verbal Behavior, 20, $470-477$

KATO, T. (1985). Semantic-memory sources of episodic retrieval failure. Memory \& Cognition, 13, 442.452.

MURDOCK, B. B., JR. (1963). Interpolated recall in short-term memory. Journal of Experimental Psychology, 66, 525-532.

Neisser, U., Hoenig, Y., \& Goldstein, E. (1969). Perceptual organization and the prefix effect. Joumal of Verbal Learning \& Verbal Behavior, 8, 424-429.

TUlving, E., \& ARBuckle, T. Y. (1963). Sources of intratrial interference in immediate recall of paired-associates. Journal of Verbal Learning \& Verbal Behavior, 1, 321-334.

WatKIns, M. J. (1979). Engrams as cue-grams and forgetting as cue overload: A cueing approach to the structure of memory. In C. R. Puff (Ed.), Memory organization and structure (pp. 347-372). New York: Academic Press.

Watkins, M. J., \& WAtkins, O. C. (1976). Cue-overload theory and the method of interpolated attributes. Bulletin of the Psychonomic Society, 7, 289-291.

WatkINS, O. C., \& WatkINS, M. J. (1975). Buildup of proactive inhibition as a cue-overload effect. Journal of Experimental Psychology: Human Learning \& Memory, 104, 442-452.

(Manuscript received February 15, 1984; revision accepted for publication March 1, 1985.) 